\title{
La Alianza del Pacífico, aspectos jurídicos organizacionales y de su sistema de solución de controversias
}

\author{
The Pacific Alliance, organizational legal \\ aspects and its dispute resolution system
}

Jaime R. Gallegos Zúñiga*

\begin{abstract}
RESUMEN
La Alianza del Pacífico ha sido abundantemente analizada por las relaciones internacionales, las ciencias políticas y la economía, poniendo de relieve algunos elementos que la caracterizan, presentándola como un fenómeno nuevo a nivel de integración en América Latina.
\end{abstract}

Sin embargo, este esquema no ha sido suficientemente abordado desde el Derecho, y haciéndonos cargo de ese vacío relativo, este trabajo busca aportar una mirada general acerca de sus aspectos orgánicos y de su sistema de solución de controversias, comparando lo planteado en esta propuesta con las experiencias sobre la materia que se han dado en otras iniciativas de integración vigentes, a fin de poder proyectar la eficiencia de sus disposiciones jurídicas.

Palabras clave: Alianza del Pacífico - Aspectos orgánicos Solución de controversias - Integración económica - Derecho comunitario.

Doctor (C) en Derecho (Universidad Complutense de Madrid). Máster en Derecho de los Negocios Internacionales y en Estudios Avanzados en Derecho Financiero y Tributarios (Universidad Complutense de Madrid). Profesor asistente del Departamento de Derecho Económico de la Universidad de Chile.jgallegos@derecho.uchile.cl. Recibido el 8 de julio de 2019. Aprobado el 7 de octubre de 2019. 


\section{ABSTRACT}

The Pacific Alliance has been abundantly analyzed by international relations, political science and economics, highlighting some elements that characterize it, presenting it as a new phenomenon at the level of integration in Latin America.

However, this scheme has not been sufficiently addressed by Law, and by taking charge of this relative vacuum, this work seeks to provide a general view of its organic aspects and its dispute resolution system, comparing what is proposed in this proposal with the experiences, on the subject, that have taken place in other initiatives of integration in force, in order to be able to project the efficiency of their legal dispositions.

Keywords: Pacific Alliance - Organic aspects - Dispute settlement - Economic integration - Community law.

\section{I.- INTRODUCCIÓN}

La Alianza del Pacífico es un bloque formado por México, Colombia, Perú y Chile, con la finalidad de obtener las ventajas generales de la integración, como son, entre otras, el aprovechamiento de economías de escala, la intensificación de la competencia, posibilidad de desarrollar actividades difíciles de emprender aisladamente, el aumento del poder de negociación y el logro de un alto nivel de empleo (Tamamés y Huerta, 2010: 215-220), en un escenario marcado por el fuerte impulso económico que están ejerciendo los países del Asia Pacífico.

Todos los Estados partes son economías marcadamente abiertas, miembros de la Asociación Latinoamericana de Integración (ALADI) y de la Organización Mundial de Comercio (омC); previamente, celebraron acuerdos de libre comercio entre sí y con
Estados Unidos, y por este motivo se dice que es un bloque afín a los intereses de esa primera potencia (Contipelli, 2017: 106-107 y Merino, 2017). Sin embargo, aún cuando en algunos casos la dependencia de ese país norteamericano es significativa, de la lectura de los objetivos perseguidos por la Alianza, cabe plantear que esta iniciativa desafía los paradigmas tradicionales del latinoamericanismo alineado con Washington y, en esta ocasión, busca proyectarse esencialmente a Asia (Thoene, Cuestas y Carmelina, 2017: 101).

La Alianza del Pacífico constituye una zona de libre comercio (zLC), ${ }^{1} \mathrm{de}$ modo análogo al Tratado de Libre Comercio de América del Norte (TLCAN), ${ }^{2}$

1 Artículo 1.1 del Protocolo Adicional (PA).

2 Artículo 101 del TLCAN. 
cuyo modelo de "regionalismo" sigue (Castaño, 2016: 299) sin crear una estructura externa conjunta, al reservarse los Estados Parte sus competencias soberanas para celebrar acuerdos con Estados ajenos al bloque, manifestación del "regionalismo abierto" de América Latina (Seatzu, 2015: 194-205).

Es un espacio de cooperación intergubernamental, carente de independencia jurídica, propuesta eminentemente práctica de sus integrantes (Oyarzún y Rojas, 2013: 20-26), que adoptan sus decisiones consensualmente. Embrión de integración regional (Thiel, 2017: 449), que evita la evaluación de los beneficios y perjuicios que el esquema reporta, $y$ el consiguiente riesgo de pretender abandonarlo.

\section{II.- Algunas CONSIDERACIONES JuRÍDiCAS ACERCA DE LA INTEGRACIÓN ECONÓMICA}

Aun cuando no es aconsejable aplicar de manera irreflexiva las teorías europeas sobre integración, que no suponen un modelo único (Arroyave, 2008: 305-306), resulta conveniente exponer las experiencias comparadas y analizarlas de acuerdo con las variables atingentes a la Alianza del Pacífico.

Así las cosas, se afirma que para que estemos ante un proceso de integración propiamente tal se requiere la creación de una nueva entidad con personalidad jurídica propia, que tenga reconocimiento internacional (Fernández Rozas y Contreras, 2013: 133-134). Así, si bien la Unión Europea cuenta con personalidad jurídica, ${ }^{3}$ esta es diferente a aquella con la que cuentan los Estados miembros, puesto que sus objetivos y funciones son específicas (Magariños, 2001: 88), es una personalidad funcional atribuida por los Estados que la integran, que

3 Artículo 47 del tue. han cedido parte de sus competencias soberanas (De Quadros, 1984: 99-103) hacia esta organización.

Atendida esta interacción, se configura un Derecho comunitario autónomo, pero integrado con los sistemas jurídicos nacionales, que no solo regula las instituciones creadas y la relación entre los Estados que participan del proceso, sino que además se caracteriza (Constanstinesco, 1970 y Pescatore, 2006: 179-270) por: i) contar con efecto directo, que implica que puede ser invocado directamente por los ciudadanos de los Estados miembros ante los tribunales u otras autoridades; ii) estar dotado de primacía por sobre las normas nacionales, y iii) ser aplicación inmediata, lo cual supone que la preceptiva comunitaria rige sin necesidad de que la normativa se internalice.

El Derecho comunitario alcanza un lugar como fuente del Derecho, en una posición normativa y jerárquica que, si bien no suprime el Derecho 
interno, sí lo condiciona, orienta y transforma (Ruiz Díaz, 2006: 629).

En el caso de la Alianza del Pacífico no se ha creado un nuevo sujeto de Derecho Internacional, dotado de personalidad jurídica, y tampoco se ha conformado una organización internacional o interestatal (Díaz-Cediel, 2016: 352), lo cual no permite diferenciar la voluntad de los Estados Parte de aquella que representa a la entidad en su conjunto. De este modo, bajo las premisas expuestas, no estaríamos ante un proceso de integración propiamente tal, que genere Derecho comunitario, y quedamos en un plano de Derecho Internacional donde, usualmente, no tiene lugar el efecto directo de los compromisos asumidos en favor de los particulares (Vajda, 2018: 205-2011), ni se verifican las demás características enunciadas.

Por otra parte, vale la pena tener en cuenta que los Estados Parte de la
Alianza del Pacífico han confirmado: i) sus derechos y obligaciones de los acuerdos internacionales en los que todos sean parte, incluido el de la Organización Mundial de Comercio, y ii) de aquellos en los que un Estado Parte y al menos otro Estado Parte sean parte, ${ }^{4}$ lo cual configura un entramado regulatorio complejo (Tremolada, 2013: 242-243), en que convergen diferentes normas, teniendo en cuenta, por ejemplo, que Colombia y Perú, simultáneamente son miembros de la Comunidad Andina de Naciones, un esquema de integración que cuenta con un acervo normativo importante, y que además coexisten diferentes acuerdos de alcance parcial de complementación económica celebrados en el marco de ALADI y numerosos tratados de libre comercio en vigor con Estados más allá de América Latina.

\section{III.- FuENTES JURÍDICAS}

\section{a) Acuerdo Marco (AM)}

El 6 de junio de 2012, en Paranal, Chile, se suscribió el sucinto y genérico (Seatzu, 2015: 202-205) Acuerdo Marco de la Alianza del Pacífico, que supuso su constitución formal y jurídica, el cual

4 Artículo 1.2 del PA entró en vigor internacional el 20 de julio de 2015. ${ }^{5}$

En su preámbulo se indica que los Estados Parte, convencidos de que la integración económica regional constituye uno de los mecanismos esenciales para que los países de América

5 Decreto № 98, de 14 de julio de 2015, del Ministerio de Relaciones Exteriores de la República de Chile. 
Latina avancen en su desarrollo económico y social sostenible, promueven que se alcance una mejor calidad de vida para sus pueblos, singularizando los objetivos perseguidos, acciones a desarrollar, algunos aspectos orgánicos, su relación con demás acuerdos y Estados, y otros elementos generales.

\section{b) Protocolo Adicional (PA)}

Con fecha 10 de febrero de 2014, se suscribió en Cartagena de Indias, Colombia, el Protocolo Adicional al citado Acuerdo Marco, el cual entró en vigor el 1 de mayo de $2016 .^{6}$

Considerando las materias abordadas (catalogadas como de cuarta generación, esto es, que no son usualmente incluidas en los tratados de libre comercio ni en las reglas multilaterales de la omc, como son: contratación pública, comercio electrónico, inversiones), se ha estimado que este Protocolo supone el acuerdo plurilateral más profundo firmado por países latinoamericanos, superando las disciplinas de ALAdI, Mercosur y la Comunidad Andina de Naciones (Bartesaghi,2016: 456, 461).

Atendido que entre los países miembros previamente contaban, entre sí, con tratados de libre comercio, el objetivo del Protocolo es armonizar y mejorar estándares comunes, especialmente en materia de disciplinas

6 Decreto № 24, del 22 de febrero de 2016, del Ministerio de Relaciones Exteriores de la República de Chile. comerciales, incorporando, además, otras temáticas no previstas en esos acuerdos, como servicios financieros y marítimos (Rebolledo, 2016: 77).

Dentro de los objetivos de este esquema ${ }^{7}$ está el construir un área de "integración profunda" para avanzar hacia la libre circulación de bienes, servicios, capitales y personas, impulsando un mayor crecimiento, desarrollo y competitividad de las economías parte, con proyección mundial y especialmente al Asia Pacífico.

Las acciones encaminadas a alcanzar ello son: ${ }^{8}$ la liberalización comercial de bienes y servicios; avanzar hacia la libre circulación de capitales y la promoción de las inversiones entre los Estados Parte; facilitación del comercio y asuntos aduaneros; promoción de la cooperación entre las autoridades migratorias y consulares; facilitación del movimiento de personas y el tránsito migratorio en el territorio de los mismos, entre otros aspectos.

De lo anterior puede desprenderse que la finalidad perseguida es la constitución de un mercado común (Bartesaghi, 2016: 462). Sin embargo, aún no se han configurado elementos hacia la estructuración de una unión aduanera que, como se sabe, conceptualmente es la fase previa dentro de los niveles de integración (tampoco se ha dicho que pretenda hacerlo), motivo por el cual algunos han planteado que debiesen emprenderse acciones

$\begin{array}{ll}7 & \text { Artículo 3.1 del AM. } \\ 8 & \text { Artículo 3.2 del AM. }\end{array}$ 
encaminadas a alcanzar tal segunda fase (Ruiz Medrano, 2017: 91).

La interpretación y aplicación del Protocolo debe hacerse conforme con los objetivos, principios y demás considerandos del Preámbulo y, de conformidad con las normas aplicables del Derecho Internacional y cada Estado Parte, debe asegurar la adopción de las medidas necesarias para dar cumplimiento a sus disposiciones en su territorio, ${ }^{9}$ precepto que podría considerarse análogo, en su medida, a un reconocimiento del principio de cooperación leal, contemplado en la Unión Europea. ${ }^{10}$

Recapitulando, aun cuando hemos afirmado que tal como hoy se presenta la Alianza del Pacífico no ha construido un Derecho Comunitario propiamente tal, si buscásemos encasillar sus fuentes normativas de acuerdo con los parámetros que en esa rama se manejan, podríamos sostener que el Acuerdo Marco y el Protocolo Adicional, con sus modificaciones, constituyen su Derecho originario, conceptualización relevante para sentar una pirámide normativa que determine la jerarquía entre los diferentes cuerpos que configuran el sistema creado.

A su turno, además de esas fuentes concretas e imperativas, ratificadas por los Congresos de los Estados Parte, se cuenta con las Declaraciones presidenciales, instrumentos de soft law, no vinculantes y de contenido meramente programático (Díaz-Cediel, 2016: 351355 y Castaño, 2016: 289).

\section{IV.- Aspectos ORgÁNicos}

Latinoamérica, aun cuando ha seguido modelos intergubernamentales, ha estructurado complejos entramados institucionales (Vargas Mazas, 2017: 79) en sus iniciativas de integración que, se estima, ha desatendido aspectos prácticos y ha caído en una burocratización excesiva (Ugarte, 2015: 27). Es por ello, y en base a tal experiencia, que la Alianza en análisis ha optado por no contar con cuerpos permanentes, siguiendo las estructuras dadas en Asia Pacífico.

9 Artículos 1.3 y 1.4 del PA.

10 Artículo 4.3 del TUE.
La instancia de decisión política de la Alianza del Pacífico son las sucesivas cumbres presidenciales que se celebran, que luego se traducen en las citadas Declaraciones, lo cual da cuenta de una estructura vertical (Rebolledo, 2016: 74), no obstante su carácter no vinculante, que se apoya en los cuerpos que a continuación se describen.

Existe un Consejo de Ministros $(\mathrm{CM}),{ }^{11}$ compuesto por los secretarios de Relaciones Exteriores y aquellos responsables del comercio exterior de los Estados Parte, el cual cuenta con

11 Artículo 4 del AM 
diferentes atribuciones. Se reúnen, de manera ordinaria, una vez al año, o extraordinariamente, a petición de uno de sus miembros, y sus acuerdos se adoptan por consenso. ${ }^{12}$

Las Decisiones aprobadas por este cuerpo y otros acuerdos convenidos en el ámbito de la Alianza del Pacífico, en desarrollo del Acuerdo Marco, son parte integrante del ordenamiento jurídico de ese bloque, ${ }^{13}$ sin que se prevean mayores precisiones acerca de la forma de incorporación de estos instrumentos en los ordenamientos jurídicos de los Estados Parte.

Se ha indicado que esta entidad es el órgano sobre el cual recae el peso de la marcha del proceso, sin que sea necesaria la participación permanente de los Jefes de Estado (Seatzu, 2015: 210).

Asimismo, se contempla ${ }^{14}$ una Comisión de Libre Comercio (CLC), compuesta por funcionarios gubernamentales de cada Estado Parte y presidida por aquél que ejerza la Presidencia pro témpore de la Alianza. Este es el cuerpo responsable de la administración del Protocolo Adicional y de su apropiada implementación.

Dentro de sus atribuciones le corresponde, entre otras: revisar el funcionamiento general del Protocolo y evaluar los resultados de su aplicación; supervisar el trabajo de los Comités, Subcomités y grupos de trabajo; contribuir en la solución de las diferencias entre los Estados Parte, ${ }^{15}$ y cumplir cualquier otra función relacionada con el Protocolo o que los Estados Parte puedan acordar.

Asimismo, le cabe, con carácter facultativo ("podrá"), emitir interpretaciones sobre las disposiciones del Protocolo. $^{16}$

Además, se prevén diferentes Grupos Técnicos formados por funcionarios de los Estados Parte, encargados de negociar disciplinas, y también Comités, que son las unidades que desarrollan el trabajo permanente del bloque. $^{17}$

A su vez, se cuenta con un Grupo de Alto Nivel (GaN), integrado por los viceministros (o subsecretarios) de Relaciones Exteriores, encargados de supervisar los reseñados grupos técnicos, evaluar las tareas en las cuales se puede progresar y preparar propuestas de relacionamiento externo.

Por último, la Secretaría es la encargada administrativa durante la Presidencia pro témpore del Estado Parte correspondiente. Esta unidad cuenta con atribuciones de organizar y ser sede de las cumbres presidenciales; coordinar las reuniones del Consejo de Ministros y del Grupo de Alto Nivel; mantener el registro de las reuniones y demás documentos; presentar al Consejo de Ministros los programas de actividades de la Alianza del Pacífico; representar a esta en los asuntos de interés común, $\begin{array}{ll}12 & \text { Artículo 5 del AM } \\ 13 & \text { Artículo 6 del AM } \\ 14 & \text { Capítulo 16 del PA. }\end{array}$
15 Artículo 17.6 del PA

16 Artículo 16.2.2 c) del PA.

17 Anexo 16.2 del PA. 
si así se lo han encargado los Estados Parte, y las demás que expresamente le confiera el aludido consejo de secretarios de Estado. ${ }^{18}$

Se ha puesto de manifiesto (Castaño, 2016: 289) el reducido rol de esta última, expresándose también (Torres, 2016: 32) que, el relacionarse externamente con otros bloques es complejo mediante tal estructura, pues si bien parece un modelo dinámico y flexible, en los hechos se da prioridad a los objetivos perseguidos por el país anfitrión, más que buscar metas que beneficien al conjunto.

En efecto, se ha planteado la inquietud de si la estructura mencionada es adecuada para los desafíos asumidos (Palacio, 2018: 20-22 y Daniels, 2015: 168-170), habida cuenta que tampoco parece aconsejable sujetar la eficacia de sus actos a la revalidación interna permanente de los Estados Parte, teniendo presente, además, que no existen procesos exitosos que se sostengan - solamente- en base a entes de cooperación intergubernamental política, como resulta ser el actual modelo de la Alianza del Pacífico (Echebarría y Estevadeordal, 2014: 41). De este modo, resultan también aplicables los reparos vertidos acerca del entramado institucional de Mercosur, en orden a que sería positivo el establecimiento de un órgano ejecutivo permanente, que actúe de modo autónomo frente a los Estados Parte y esté dotado de una "voluntad comunitaria imparcial" (Cassagne, 1992: 362-363).

Asimismo, un vacío sensible -al igual que ocurre en Mercosur y en el TLCAN- es la carencia de un efectivo Tribunal de Justicia Permanente, que resuelva de modo imparcial los conflictos que se susciten, dando aplicación e interpretando la normativa comunitaria.

\section{V.- Solución de controversias}

\section{a) Reseña general}

\section{El Capítulo 17 del Protocolo Adicional} es el concerniente a Solución de Diferencias relativas a la interpretación, implementación o aplicación de las disposiciones del PA o cuando un Estado Parte considere que: (a) una medida vigente o en proyecto de otro EP

18 Artículo 7 del AM. es o pudiera ser incompatible con las obligaciones previstas en el PA; (b) un EP ha incumplido de otra manera con las obligaciones previstas en el PA, o (c) una medida vigente o en proyecto de otro EP cause o pudiera causar anulación o menoscabo de los beneficios que razonablemente pudo haber esperado recibir de la aplicación del PA. ${ }^{19}$

19 Artículo 17.3 del PA. 
Cada Estado Parte, "en la medida de lo posible", debe notificar a los otros cualquier medida en proyecto o vigente que considere que pudiera afectar materialmente el funcionamiento del Protocolo Adicional, o que sustancialmente afecte los intereses de otro EP conforme al mismo, y establecer o mantener tribunales o procedimientos judiciales, cuasi-judiciales o administrativos, con el propósito de una pronta revisión y, cuando se justifique, la corrección de las acciones "administrativas" definitivas relacionadas con asuntos comprendidos en el PA. Tales tribunales deben ser imparciales e independientes de la autoridad encargada de aplicar las medidas y no tener ningún interés sustancial en el resultado del asunto. ${ }^{20}$

De esta manera, se establece una vía internacional/comunitaria para resolver las controversias del Capítulo 17 del Protocolo, y una interna, que parecería dar lugar a una aplicación directa del PA en favor de particulares que quisieren impugnar una medida administrativa que contraviniese el ordenamiento del PA, con los reparos que se mencionarán más adelante.

Luego, debe precisarse que no se prevé la posibilidad de impugnar las actuaciones de los órganos de la AP, ni por los EP (no tendría sentido, habida cuenta de la regla de adopción de decisiones de modo consensual), ni por los particulares y, en su caso, a estos últimos solo les restaría acudir a las instancias locales con competencia sobre los funcionarios que integren esos cuerpos.

Por otro lado, se señala que la regulación prevista en ese Capítulo 17 busca proporcionar un efectivo, eficiente y transparente proceso de solución de diferencias. ${ }^{21}$

Sobre este punto, uno de los más objetados del mecanismo de solución de controversias del tLCAN dice relación con su falta de transparencia (Ortíz, 2002: 28-29). Otros, en cambio, elogian la confidencialidad con que se desarrolla, en el entendido que con ello se velaría por la imparcialidad de los miembros del panel (Picker, 1997: 527-528)

El procedimiento del Capítulo 17 del PA consta de tres fases, una primera de consultas, una segunda de intervención de la Comisión de Libre Comercio y una final (eventuales, si no se ha podido resolver previamente la disputa) de arbitraje, mediante paneles ad hoc, establecidos para resolver la diferencia puntual, de modo similar al contemplado en el mecanismo de la омс, que no describiremos y daremos por conocido, concentrándonos, en lo sucesivo, en exponer algunos temas que pudieren resultar controvertidos. 
b) Asuntos que pueden resultar controvertidos

\section{B. I) LEGITIMADOS ACTIVOS}

Teniendo a la vista que el Protocolo Adicional sigue el modelo del TLCAN, se infiere que la legitimación queda limitada a los Estados Parte del acuerdo (Hansen, 2003: 490), sin embargo, han surgido reparos a esta lectura (Thiel, 2017: 452-454).

En esquemas equiparables se ha sostenido que, al no conferírsele la posibilidad de accionar directamente a los privados, se amplía la discrecionalidad de los Estados, politizando el régimen de solución de conflictos, según la afinidad que tenga el particular con su Estado de domicilio (Whitelaw, 2004: 147 y Perotti, 2008: 55), y en un caso concreto, un importador, por ejemplo, no podría cuestionar, mediante el sistema supralocal o de integración, la juridicidad de una medida adoptada por el propio Estado donde tenga asiento, y solo le cabe acudir a los procedimientos internos correspondientes, con el riesgo de que analice la cuestión bajo un prisma local y no uno comunitario, lo cual afecta el funcionamiento del sistema como un todo, o lo coloca en una situación de indefensión (Jovtis, 2012: 89-93).

Otra duda cabe respecto del alcance que debe dársele al término tercero, si solo comprende a aquellos Estados signatarios de los instrumentos de la Alianza del Pacífico, los Estados observadores o si incluso comprendería a otros ajenos a este esquema, atendida la vocación universal y abierta de este sistema subregional (Thiel, 2017: 455-457).

A su vez, al no existir un ente autónomo (regla de la unanimidad) comunitario que vele por los intereses del bloque en su conjunto, no existe la posibilidad de que un órgano de la Alianza del Pacífico persiga el incumplimiento de obligaciones asumidas por los Estados Parte, de modo análogo a las competencias de la Secretaría General de la Comunidad Andina de Naciones y de la Comisión en la Unión Europea. ${ }^{22}$

\section{B.2) POSIBILIDAD DE ACUDIR A}

\section{DIFERENTES FOROS}

Las diferencias sobre un mismo asunto relacionadas con lo dispuesto en el Protocolo Adicional, ${ }^{23}$ en el Acuerdo de la omc o en cualquier otro convenio comercial del que los EP sean parte, pueden resolverse en cualquiera de dichos foros, a elección del reclamante.

Luego, bajo la lógica del principio de prevención, elegido y radicado el asunto en uno de los foros, pierden competencia potencial los demás.

Esta opción de foro ha sido criticada, puesto que le resta eficacia al sistema establecido en la Alianza, dando pie a que su mecanismo sea solo una alternativa adicional para zanjar

22 Artículos 23 del Tratado de Creación del TJCAN, y 258 tFue.

23 Artículo 17.4 del PA. 
disputas (Thiel, 2017: 457-459), configurando un sistema de membresías superpuestas, donde la elección del foro se dirimirá según si se pretende sentar un precedente regional o multilateral (Busch,2007: 735-761), atendiendo a los plazos previstos para la solución de los litigios, la obligatoriedad de lo fallado o la existencia de recursos para impugnar lo decidido, entre otros elementos.

Como ya se señaló, este modelo sigue los lineamientos del TLCAN, cuyo mecanismo general de solución de diferencias, del Capítulo XX, ha sido pocas veces empleado, puesto que las partes signatarias de ese acuerdo han preferido acudir al sistema de la омC (Vidigal, 2017: 930 y Fattore, 2018: 87).

Debe tenerse en cuenta que en aquellos esquemas en que se ha alcanzado mayores niveles de integración, como en la Unión Europea, se establecen foros exclusivos para conocer los asuntos, ${ }^{24}$ lo cual ayuda a la formación de jurisprudencia coherente (Henckels, 2008: 572-573).

Sobre la opción de foro, se ha dicho que los países en vías de desarrollo, ante la alternativa de deducir las reclamaciones mediante un instrumento regional o el sistema de la omc, muchas veces son reacios a presentarlas en el segundo, por los costos y mayor capacidad jurídica que demanda afrontar un asunto en Ginebra (Fattore, 2018: 82-85 y Davis, 2009: 26).

Con todo, el sistema de la omc puede resultar más efectivo que algunos mecanismos de los tratados de libre comercio, puesto que, por ejemplo, bajo el tLCAN lo resuelto por los paneles no resulta efectivamente exigible (Álvarez y Beltrán, 2018: 237), mientras que en el sistema multilateral suele producirse una presión internacional de los restantes Estados que integran esa organización para que el Estado denunciado acate lo dictaminado por el panel respectivo.

Todo lo anterior, sin perjuicio de la crisis por la cual está pasando actualmente el sistema multilateral de comercio y en particular su mecanismo de solución de controversias.

\section{B.3) SELECCIÓN DEL TRIBUNAL ARBITRAL}

El tribunal se integra por tres árbitros. Cada parte en la diferencia, dentro de 20 días, contados a partir de la recepción de la solicitud de establecimiento del tribunal, debe designar un árbitro, quien "puede ser" su nacional y, además, debe proponer hasta cuatro candidatos para actuar como su presidente, quien no puede ser nacional ni tener su residencia permanente en alguno de los Estados Parte en conflicto.

No se contempla una nómina permanente de sujetos susceptibles de ser designados árbitros, lo cual supone una marcada diferencia con lo establecido en Mercosur, donde sí se cuenta

24 Artículo 344 del tfue. 
con esa lista estable, ${ }^{25}$ que se compone de 12 individuos que cada Estado miembro ha designado y registrado ante la Secretaría del bloque, elemento que - a nuestro juicio- confiere mayor certeza en este tipo de asuntos.

Retomando el Protocolo Adicional, si un Estado parte no designa árbitro dentro del plazo mencionado, este es seleccionado por el otro de la lista indicativa de expertos que pueden ser integrantes de grupos especiales de la omc de la parte que no designó, y en caso de que los candidatos de esa nómina no estuvieran disponibles, se debe seleccionar entre los miembros de ese mismo registro de cualquiera de las Partes, distintos a las de la diferencia ${ }^{26}$.

Con esta regulación se castiga la desidia en nombrar los árbitros que conocerán el asunto, puesto que, de presentarse esta circunstancia, se habilita para que su contraparte designe al juzgador que a ella le correspondiere nombrar, a diferencia de lo contemplado en otros esquemas en que esta decisión se le encomienda a un tercero, generalmente por sorteo ${ }^{27}$.

Las partes en la diferencia deben designar, de común acuerdo, al presidente del tribunal entre los candidatos propuestos. Si vencido el plazo no hay acuerdo, la Presidencia Pro Tempore, en

25 Artículos 10 y 11 del Protocolo de Olivos $(\mathrm{PO})$ para la solución de controversias en el Mercosur.

26 Artículo 17.13.3 del PA.

27 Artículos 10.2.ii) del PO y 2011.1.d) del TLCAN. un plazo de siete días, lo escoge por sorteo. Esto plantea la inquietud acerca de qué ocurre si es precisamente ese EP el que es denunciado por infringir el PA y pretende bloquear la formación del tribunal.

En el trCan la elección del panel debe efectuarse atendiendo a la nómina que tenía que conformarse, ${ }^{28}$ la cual no se materializó, haciendo más difícil detectar potenciales conflictos de interés, retrasando las designaciones en su momento y, en definitiva, bloqueando su conformación, por lo cual el sistema ha dejado de estar operativo (Lester, Manak y Arpas, 2019: 64-66).

Así las cosas, parece positivo que la Alianza del Pacífico haya intentado evitar se produzcan obstrucciones en esta materia, con la salvedad dicha acerca del sorteo de la presidencia pro témpore.

\section{B.4) CARACTERÍSTICAS DEL LAUDO}

En el laudo final, según se indica, es definitivo, inapelable y "obligatorio" para las partes de la diferencia, ${ }^{29}$ y en él se deben plasmar, de ser el caso, las opiniones divergentes sobre asuntos en los que no haya habido una decisión unánime ${ }^{30} \sin$ que se revele la identidad de los árbitros que votaron con la mayoría o minoría. ${ }^{31}$

\footnotetext{
28 Artículos 2011 y 2009 del tLCAN.

29 Artículo 17.16.2 del PA.

30 Artículo 17.16.1 del PA.

31 Artículo 17.16.4 del PA, disposición equivalente a los artículos 2017.2 del TLCAN y 14 del ESD.
} 
En Mercosur ${ }^{32}$ — de modo análogo a lo que ocurre en la омс- se prevé un mecanismo del tipo casación (su examen se ciñe a cuestiones de Derecho), que se le denomina revisión. Distinta es la situación en el TLCAN, donde no se establecen recursos contra los laudos emitidos, lo cual ha sido reprochado por la doctrina (Sher, 2003: 1021).

Se ha sostenido que, con un sistema de apelación dentro de espacios de integración, se camina hacia la unificación de jurisprudencia (Susani, 2010: 75, 80), más aún si se cuenta con un órgano permanente encargado de revisar esta clase de asuntos (Opertti, 2004: 157).

En la Unión Europea, la Comunidad Andina y el Mercosur, ${ }^{33}$ en aras a la construcción de un Derecho comunitario, con una sola voz, que procure asegurar la independencia de los jueces (Isaac, 2000: 330-331 y Pollack, 2018: 161), la posibilidad de consignar votos de minoría ( $\mathrm{y} / \mathrm{o}$ fundamentarlos) se encuentra excluida, asunto que, por motivos de publicidad y enriquecimiento de los pronunciamientos, paulatinamente está siendo reprochado

32 Artículo 17 del PO.

33 Artículos 35 del Estatuto del tjue y 27 del Reglamento de Procedimiento ante el TJE (de modo indirecto en el caso europeo); 90 del Estatuto del TJCAN, 25 del PO, acerca de los laudos emitidos. Sobre el particular, debe hacerse presente que en Mercosur se presenta la paradoja de que en las opiniones consultivas sí se admite consignar las opiniones de disidencia (artículo 9 del Reglamento del PO).
(Azizi, 2011: 49-68 y Morales, 2018: 74-94).

\section{B.5) CUMPLIMIENTO DEL LAUDO}

Una vez notificado el laudo final del tribunal arbitral, las partes en la diferencia deben "llegar a un acuerdo sobre su cumplimiento" en los términos de las determinaciones, conclusiones y "recomendaciones" del tribunal arbitral. $^{34}$

Cuando el laudo determine que la medida es incompatible con las disposiciones del Protocolo o que una medida de esa parte es causa de anulación o menoscabo, el reclamado debe, "siempre que sea posible", eliminar el incumplimiento, anulación o menoscabo, ${ }^{35}$ terminología que le resta fuerza al veredicto emitido.

Los pronunciamientos son declarativos y no tienen efecto directo, pues no ordenan ni mandatan cumplir nada en particular, y simplemente se limitan a constatar una infracción a la normativa, a la espera de un cumplimiento prospectivo, más que la corrección de infracciones en las que se ha incurrido, sin que tampoco se logren reparaciones por el daño causado con la medida ilegítima.

En el TLCAN se han criticado las demoras en los cumplimientos a lo resuelto por los paneles (por ej., en el caso de transportes de carga (USA98-2008-01), cuya implementación se

34 Artículo 17.19.1 del PA.

35 Artículo 17.19.2 del PA. 
extendió por más de 2 años), como también el rechazo de su ejecución por parte de las cortes locales (Sher, 2003: 1001-1026), atendido el carácter no vinculante de las decisiones emitidas, ${ }^{36}$ que conlleva a constatar que el mecanismo estructurado cuenta con un bajo nivel de legalización (Steger, 2004: 196).

En Mercosur se indica ${ }^{37}$ que los laudos son obligatorios para los Estados Parte en la controversia y cuentan, con relación a ellos, con fuerza de cosa juzgada, debiendo ser cumplidos en la forma y con el alcance con que fueron dictados. La adopción de medidas compensatorias no exime al Estado reclamado de su obligación de cumplir el laudo. ${ }^{38}$

Ahora bien, conviene advertir que aun cuando se constate una violación a la normativa del Mercosur, ello no implica que el pronunciamiento tenga efectos anulatorios por sí mismo, lo que ha generado que tanto en ese bloque, como en la Comunidad Andina de Naciones, se exhiban incumplimientos sintomáticos de los laudos y sentencias emitidas (Alonso, 2008: 91-92).

\section{B. 6) COMPENSACIÓN O SUSPENSIÓN} DE BENEFICIOS

Si las partes no llegan a un acuerdo sobre el cumplimiento del laudo o una solución mutuamente satisfactoria de la diferencia, dentro de los 30 días siguientes a la notificación de dicho pronunciamiento, la parte reclamada, a solicitud de la reclamante, debe iniciar negociaciones a fin de establecer una compensación mutuamente aceptable. Tal compensación debe ser temporal y otorgada hasta que la diferencia se solucione. ${ }^{39}$

Si no se logra acuerdo acerca del cumplimiento ni de la compensación aludida (dentro de los 30 días siguientes), el reclamante queda habilitado para suspender a la parte reclamada beneficios y otras obligaciones previstas en el Protocolo, medidas que pueden ser revisadas por el mismo tribunal arbitral que conoció del asunto principal. $^{40}$

Sobre este punto debe tenerse en cuenta que, si bien la Alianza del Pacífico sigue los lineamientos del Entendimiento de Solución de Diferencias de la Organización Mundial de Comercio, ${ }^{41}$ los plazos del Protocolo Adicional, para suspender los beneficios y requerir compensación, son bastante más breves, puesto que en el

\footnotetext{
39 Artículo 17.20.1 del PA.

40 Artículos 17.20.2 y 17.22 del PA.

41 Artículo 22 del ESD.
} 
plano multilateral no deben exceder los 15 meses.

En lo que se refiere a retorsiones, se ha afirmado que, dentro del marco del TLCAN, resulta más complejo conminar a un país de mayor peso relativo para que modifique su normativa cuestionada que, si el asunto se ventila en el marco de la omc, donde se puede alcanzar la presión de diferentes miembros de ese cuerpo multilateral (Vidigal, 2017: 927-950 y Ortiz, 2002: 20-30).

La retorsión, como contramedida institucionalizada, supone una ejecución descentralizada basada en la retaliación, que deja a las propias partes la implementación de lo resuelto, facilitando que, aquella con mayor poder relativo, adopte una decisión en favor de sus intereses, todo lo cual juega contra el funcionamiento de un sistema de integración (Carvalho de Vasconcelos, 2016: 131-132), pues supone la autorización para incumplimientos mutuos que merman el libre comercio y el mercado común que se pretende alcanzar, y coloca en una compleja posición al país reclamante, por lo cual debe preferirse el otorgamiento, en primer término, de concesiones, lineamiento que parece seguir el Protocolo Adicional.

Siguiendo la lógica recién expuesta, en la Unión Europea no se establecen medidas compensatorias o contramedidas para coaccionar la ejecución de lo resuelto por el Tribunal de Justicia de la Unión Europea (Pastori, 2006: 72), y para instar el cumplimiento de lo decidido - que tiene un carácter más que meramente declarativo (Giménez, 2004: 70-80) — se pueden aplicar sanciones pecuniarias ${ }^{42}$ que han resultado ser bastante efectivas (Díez-Hochleitner, 1993).

\section{B.7) INTERPRETACIÓN UNIFORME DE LA NORMATIVA}

Las posibilidades de alcanzar una interpretación uniforme y armónica de la normativa comunitaria pueden lograrse con órganos especializados que emitan pronunciamientos obligatorios, mediante figuras como las cuestiones e interpretaciones prejudiciales de la Unión Europea y la Comunidad Andina de Naciones, respectivamente, y, en menor medida, las opiniones consultivas (no obligatorias) del Mercosur.

En la Alianza del Pacífico no se ha contemplado un cuerpo jurisdiccional que ejerza ese rol, y solo cabe indicar que la Comisión de Libre Comercio está habilitada para emitir interpretaciones acerca del Protocolo Adicional, ${ }^{43}$ pero no se precisa cómo debe ejercer esa función, y tampoco se norman los efectos de esta facultad en el plano comunitario o en procedimientos judiciales o administrativos

42 Artículo 260 del true. Además, los particulares podrían perseguir, ante sus propios tribunales nacionales, la responsabilidad del Estado correspondiente, buscando se resarzan los perjuicios que esa conducta conlleva.

43 Artículo 16.2.2.c) del PA. 
internos, como sí se configura en el TLCAN ${ }^{44}$ y en los citados bloques subregionales.

De este modo, la Alianza del Pacífico renuncia a crear un Derecho comunitario y establece un sistema de solución de controversias que no se presenta como efectivo para resolver la disputa misma y que tampoco parece estar encaminado a conferir certeza mediante una interpretación coherente y armónica de los distintos laudos que sucesivamente se vayan dictando por árbitros, que no tienen continuidad ni permanencia en el conocimiento de los asuntos.

\section{VI.- Consideraciones finales}

La Alianza del Pacífico presenta carencias como proceso de integración y en la conformación de un Derecho Comunitario (Seatzu, 2015: 204-205), sin que, por ejemplo, se brinde - de modo estable y formal - la posibilidad de interactuar (proponiendo o impugnando) entre los ciudadanos de los Estados Parte y los órganos del esquema. Tales vacíos no parecen hacer viable la concreción de los ambiciosos objetivos de integración profunda planteados en el Acuerdo Marco. ${ }^{44}$

$\mathrm{Su}$ avance es $\tan$ incipiente que no cabe ponderar los principios de efecto directo, aplicación inmediata y supremacía de una manera clara, y algunas disposiciones aisladas solo permiten conjeturar eventuales interpretaciones que pudieran darse de sus normas.

El sistema de solución de controversias si bien, en determinados ámbitos, recoge la experiencia de otros acuerdos, presenta deficiencias advertidas en el funcionamiento del TLCAN y del Mercosur, lo cual nos conduce a un escenario de incertidumbre, pues su mecanismo se ve poco atractivo, y el de la омc pasa por momentos complejos, atendido lo cual, de incrementarse los flujos y transacciones dentro de la Alianza, no se cuenta con un sistema eficiente que pueda resolver los conflictos que naturalmente pudieren suscitarse y esta es una temática que debiere abordarse por los actores encargados de impulsar el proyecto.

44 Artículo 2020 del trcan. 


\section{Bibliografía}

Alonso, R. (2008). Un paseo por la jurisprudencia supranacional europea y su reflejo en los sistemas suramericanos de integración, Córdoba: Advocatus.

Álvarez, J.y Beltrán, D. (2018). “The Pacific Alliance Dispute Settlement Mechanism: One More for the Heap", en Sauvé, Pierre et al, (eds.) The Pacific Alliance in a World of Preferential Trade Agreements Lessons in Comparative Regionalism. Cham: Springer, pp. 235-249.

Arroyave, M. (2008). “Ineficacia crónica del Derecho andino”. Papel Político, vol. 13, № 1, pp. 299-321.

Azizi, J. (2011). “Unveiling the EU Courts' Internal Decision-Making Process: A Case for Dissenting Opinions?". Era Forum, vol 12, № 1, pp. 49-68.

Bartesaghi, I. (2016). "Los desafíos de la Alianza del Pacífico en el marco del TPP”, en Tremolada, Eric (ed.) El Derecho internacional y su influencia en las ciencias constitucional y económica modernas. Bogotá: Universidad Externado, pp. 451-469.

Busch, M. (2007). "Overlapping Institutions, Forum Shopping, and Dispute Settlement in International Trade". International Organization, vol. 61, № 4, pp. $735-761$.

Carvalho De Vasconcelos, R. (2016). "O Mercosul e as linhas gerais de seu sistema de solução de controversias". Revista de la Secretaría del Tribunal Permanente de Revisión, vol. 4, № 8, pp.113-135.

Cassagne, J.C. (1992). "El Mercado Común del Sur: Problemas jurídicos y organizativos que plantea su creación”. Revista de Administración Pública № 127, pp. 351-365.

Castaño, J. (2016). "Análisis y perspectivas de la Alianza del Pacífico". Estudios de Deusto, vol. 64, no 1, pp. 281-305.

Constantinesco, L.J. (1970). L' applicabilité directe dans le droite de la C.E.E. París: Librairie Genérale de Droit et Jurisprudence.

Contipelli,E. (2017). “Gobernanza global y análisis comparado de los procesos de integración en América Latina: Comunidad Andina y el Mercado del Sur".Revista de Relaciones Internacionales, Estrategia y Seguridad, vol.12, № 2, pp. 93-110. Daniels, Ch. (2015). “The Pacific Alliance and Its Effect on Latin America: Must a Continental Divide be the Cost of a Pacific Alliance Success?”. The Loyola of Los Angeles International \& Comparative Law Review, vol. 37, № 2, pp. 153-183.

Davis, Ch. (2009). "Overlapping Institutions in Trade Policy". Perspectives on Politics, vol. 7, N 1, pp. 25-31.

De Quadros, F. (1984). Direito das Comunidades Europeias. Lisboa: Almedina.

Díaz-Cediel, S. (2016). "La Alianza del Pacífico: un escrutinio jurídico al tenor del Derecho de las organizaciones internacionales". Anuario Colombiano de Derecho Internacional, vol.9, pp.347-390. Díez-Hochleitner, J. (1993). "La respuesta del TUE al incumplimiento de las sentencias del Tribunal de Justicia por los Estados miembros". Revista de Instituciones Europeas, vol. 20, № 3, pp. 837-899. 
Echebarría, L. y Estevadeordal, A. (2014). "Alianza del Pacífico: Un nuevo motor de integración regional”, en Foxley, A.y Meller, P. (eds.) Alianza del Pacifico: En el proceso de integración latinoamericana. Santiago: CIEPLAN, pp. 27-42.

Fattore, Ch. (2018). "Forum Shopping and Fairness in Mexico-United States Trade Disputes”. Latin American Policy, vol. 9, № 1, pp. 77-93.

Fernández Rozas, J.C.y Contreras, L. (2013). Derecho del comercio internacional y polí tica comercial de la República Dominicana. Santo Domingo: Funglode.

Giménez, I. (2004). La eficacia de las sentencias dictadas por el tJCE. España: Cizur Menor y Thomson Aranzadi.

Hansen, P. (2003)."Judicialization and Globalization in the North American Free Trade Agreement". Texas International Law Journal, vol. 38, № 3, pp. 489-503.

Henckels, C. (2008). “Overcoming Jurisdictional Isolationism at the WTO-FTA Nexus: A Potential Approach for the WTO". The European Journal of International Law, vol. 19, №3, pp. 571-599.

Isaac, G. (2000). Manual de Derecho Comunitario General, 5ta ed. Barcelona: Ariel. Jovtis, I. (2012). “La legitimación activa en el sistema de solución de diferencias del Mercosur", en Hernández, M. et al (dirs.), Tribunales en Organizaciones de Integración: Mercosur, Comunidad Andina y Unión Europea. España: Cizur Menor y Thomson Reuters Aranzadi, pp. 87-100.

Lester, S.; Manak, I. \& Arpas, A. (2019). "Access to Trade Justice: Fixing NAFTA's Flawed State-to-State Dispute
Settlement Process". World Trade Review, vol. 18, $\mathrm{N}^{\circ} 1$, pp. 63-79.

Magariños, G. (2001). "El Derecho de integración en la ALADI”, en CAN (ed.) Integración y Supranacionalidad. Soberanía y Derecho Comunitario en los países andinos. Lima: Secretaría General de la Comunidad Andina, pp. 85-97.

Merino, G. (2017). “Proyectos estratégicos e integración regional en América Latina. El surgimiento de la Alianza del Pacífico, el fortalecimiento del regionalismo abierto y el retroceso del regionalismo autónomo". Relaciones Internacionales № 52, pp. 17-37.

Morales, M. (2018). “Los votos particulares y las disidencias en los tribunales internacionales confrontados al principio de publicidad. Un desafío para la Corte de Luxemburgo". Revista de Estudios Europeos № 72, pp. 74-94.

Opertti, D. (2004). "El nuevo mecanismo de solución de controversias del Mercosur", en Lacarte, J. y Granados, J. (eds.), Solución de controversias comerciales intergubernamentales: Enfoques multilaterales y regionales. Buenos Aires: INTAL, pp. 149-158.

Ortiz, A. (2002). “La solución de controversias en el TLCAN: un esbozo sobre su desempeño y retos”. Arbeitspapiere des IIK № 2, pp. 1-33.

Oyarzún, L. y Rojas, F. (2013). “La Alianza del Pacífico en América Latina ¿Contrapeso regional?". Cuadernos sobre Relaciones Internacionales, Regionalismo y Desarrollo, vol. 8, № 16, pp. 9-30.

Palacio, A.M. (2018). "The Pacific Alliance: Building a Pathway to the 
High-Hanging Fruits of Deep Integratio", en Sauvé, P. et al, (eds.) The Pacific Alliance in a World of Preferential Trade Agreements Lessons in Comparative Regionalism. Cham: Springer, pp. 3-27.

Pastori, A. (2006). "La ejecución de las sentencias y laudos en la integración regional”. Cuadernos de Integración Europea, № 5, pp. 61-93.

Perotti, A. (2008). Tribunal Permanente de Revisión y Estado de Derecho en el Mercosur. Buenos Aires: Marcial Pons.

Pescatore, P. (2006). L' ordre juridique des Communautés Européennes. Étude des sources du droit communautaire (reimpresión). Bruselas: Bruylant.

Picker, S. (1997). “The NAFTA Chapter 20 Dispute Resolution Process: A view from the Inside". Canada-United States Law Journal, vol. 23, pp. 525-540.

Pollack, M. (2018). "The legitimacy of the European Court of Justice", en Grossman, N.et al (eds.) Legitimacy and International Courts. Cambridge: Cambridge University Press, pp. 143-173.

Rebolledo, A. (2016). "El Acuerdo Marco y el Protocolo Adicional de la Alianza del Pacífico. Visión desde Chile”. Revista Mexicana de Política Exterior $\mathrm{N}^{\circ} 106$, pp. 67-85.

Ruiz Díaz, R. (2006). “Las opiniones consultivas ante el Tribunal Permanente de Revisión del Mercosur a través de los tribunales superiores de los Estados parte”. Anuario de Derecho Constitucional Latinoamericano, tomo I, pp. 629-651.

Ruiz Medrano, S. (2017). "La Alianza del Pacífico. Una oportunidad más para la Integración de Latinoamérica. Aspectos
Políticos y Jurídicos”, en Mora, J. y Osorio-Caballero, M. (comp.) La Alianza Del Pacífico: Nuevos retos e implicaciones para Latinoamérica. Cali: Javieriano, pp. 81-100.

Seatzu, F. (2015). "The Alliance of the Pacific: A new instrument of Latin American and Caribbean Economic Integration?", en Odello, M. y Seatzu, F. (eds.), Latin American and Caribbean International Institutional Law. La Haya: Springer, pp. 193-218.

Sher, M. (2003). "Chapter 20 Dispute Resolution under NAFTA: Fact or Fiction”, George Washington International Law Review, vol.35, № 4, pp 1001-10026.

Steger, D. (2004). "La solución de controversias en el Tratado de Libre Comercio de América del Norte”, en Lacarte, J. y Granados, J. (eds.), Solución de controversias comerciales intergubernamentales: Enfoques multilaterales y regionales. Buenos Aires: INTAL, pp. 187-202.

Susani, N. (2010). "Dispute Settlement", en Toscano, M. et al (eds.) The Law of Mercosur. Oxford: Hart Publishing, pp. 73-85.

Tamamés, R. y Huerta, B. (2010). Estructura económica internacional, 21a edición. Madrid: Alianza Editorial.

Thiel, M. (2017). “Alliance du Pacifique: enjeu de l'esthétique de la résolution des différends". Anuario Mexicano de Derecho Internacional, vol. 17, pp. 443-467.

Thoene, U.; Cuestas, E. y Carmelina, M. (2017).“La Alianza del Pacífico y el regionalismo latinoamericano: en búsqueda de una revitalización autonomista de 
la integración”. Análisis Político № 89, pp. 91-110.

Torres, M. (2016). “The New Model of Regional Integration for Emerging Countries in Latin America: The Pacific Alliance", en Torres, M. y Violante, J. (eds.) Emerging Markets. The Pacific Alliance Perspectives \& Opportunities for Latin America. Salamanca: European Institute of International Studies, pp.19-44. Tremolada, E. (2013). "La Alianza del Pacífico: ¿una organización consecuente con sus objetivos?", en Tremolada, Er. (ed.), Repensando la integración y las integraciones. Bogotá: Universidad Externado de Colombia, pp. 235-258.

Ugarte, M. (2015). “La configuración de un nuevo marco jurídico de integración de la Alianza del Pacífico". Gestión en el Tercer Milenio № 16, pp. 15-41.
Vajda, Ch. (2018). "The EU and Beyond: Dispute Resolution in International Economic Agreements". The European Journal of International Law, vol. 29, № 1, pp. 205-224.

Vargas-Mazas, E. (2017). "L’Alliance du Pacifique et le discours néolibéral du développement". Cahiers des Amériques Latines № 85, pp. 71-90.

Vidigal, G. (2017). "Why is there so little litigation under Free Trade Agreements? Retaliation and Adjudication in International Dispute Settlement”.Journal of International Economic Law, vol. 20, № 4, pp. 927-950.

Whitelaw, J. (2004). "Régimen de solución de controversias en el Mercosur", en Lacarte, J.y Granados, J. (eds.), Solución de controversias comerciales intergubernamentales: Enfoques multilaterales y regionales. Buenos Aires: INTAL, pp.139-147. 